\title{
Trabeculectomy with versus without releasable sutures for glaucoma: a meta-analysis of randomized controlled trials
}

\author{
Minwen Zhou ${ }^{\dagger}$, Wei Wang ${ }^{\dagger}$, Wenbin Huang and Xiulan Zhang ${ }^{*}$
}

\begin{abstract}
Background: The aim of this study was to compare the efficacy and tolerability of trabeculectomies performed with and without releasable sutures in the treatment of patients with uncontrolled glaucoma.

Methods: A comprehensive literature meta-analysis was performed, comparing trabeculectomies performed with and without releasable sutures. The primary efficacy measure was the weighted mean difference (WMD) in percentage intraocular pressure reduction (IOPR\%) at the follow-up end point. The secondary efficacy measure was the risk ratio $(R R)$ for complete and qualified success rates of trabeculectomy at the follow-up end point. Trabeculectomy tolerability estimates were measured by the RR for adverse events. All the outcomes were reported with a $95 \%$ confidence interval (Cl).

Results: The WMD of the IOPR\% from baseline was -4.56 (range -9.24-0.12) when trabeculectomies without releasable sutures were compared with trabeculectomies with releasable sutures. Trabeculectomies with releasable sutures were associated with numerically greater, but nonsignificant, efficacy in terms of lowered IOP compared with trabeculectomies without releasable sutures. The complete and qualified success rate of the two surgical procedures were comparable, with RRs of 0.92 (range 0.80-1.04) and 0.99 (range 0.89-1.11), respectively, at the follow-up endpoints. Trabeculectomies without releasable sutures were associated with a significantly higher frequency of hypotony and flat anterior chambers than trabeculectomies with releasable sutures, with pooled RRs of 4.04 (range 1.88-8.68) and 2.57 (range 1.25-5.30), respectively.

Conclusion: Although the two surgical procedures resulted in equivalent efficacy in IOP control, the trabeculectomies performed with releasable sutures were better tolerated than those without releasable sutures.
\end{abstract}

Keywords: Releasable sutures, Trabeculectomy, Meta-analysis

\section{Background}

Glaucoma is a major eye disease that tends to result in blindness, and trabeculectomy is currently considered an effective surgical procedure to treat uncontrolled glaucoma $[1,2]$. Although trabeculectomy has been used for the treatment of glaucoma in clinical practice for over 40 years [3], several postoperative complications are associated with this procedure. These include early postoperative excessive filtration, which leads to a shallow or a flat anterior chamber, hypotony, and choroidal detachment [4-6].

\footnotetext{
*Correspondence: zhangx12@mail.sysu.edu.cn

'Equal contributors

Zhongshan Ophthalmic Center, State Key Laboratory of Ophthalmology, Sun Yat-Sen University, 54S.Xianlie Road, Guangzhou 510060, People's Republic of China
}

\section{() Biomed Central}

Trabeculectomy combined with releasable suture surgery has recently been used to treat uncontrolled glaucoma [7-14]. This technique aims to eliminate or minimize the complications of trabeculectomy without releasable sutures. The use of releasable sutures in trabeculectomy surgery, first mentioned by Schaffer [15], allows the surgeon to close the sclera flap relatively tightly intraoperatively, thereby helping to control the aqueous outflow and to reduce the likelihood of the aforementioned complications. When the postoperative intraocular pressure (IOP) is elevated and decreases after massage, the sutures can be removed to increase the aqueous outflow. This technique is used in clinical practice today. Laser suture lysis can also be used to lower the postoperative IOP, and reduce 
the likelihood of complications [16]. However, there is a possibility that laser suture lysis, even at a low dose, may cause burns and result in inflammation of the conjunctiva. Laser suture lysis may also lead to conjunctival scars and a flat bleb [17].

Several published randomized controlled trials (RCTs) have compared the efficacy and complications of trabeculectomies performed with and without releasable sutures $[8,11,12,14,18,19]$. However, these studies included only a modest or a small sample size, and their results were inconclusive. Therefore, to assess the efficacy and complications of these two surgical procedures for the management of uncontrolled glaucoma, we undertook a meta-analysis of all available RCTs.

\section{Methods}

This meta-analysis was performed according to a predetermined protocol, which is described below. Additionally, standard systematic review guidelines, as outlined by the Cochrane Handbook for Systematic Reviews of Interventions [20], were followed at all stages of the process.

\section{Literature search}

Four electronic databases (PubMed, ISI Web of Science, EMBASE, and the Cochrane Library) were searched systematically for studies published before September 1, 2013. The structured search strategies used the following search terms: "glaucoma" or "trabeculectomy" or "filtration surgery" or "filtering surgery" or "filtration operation" or "filtering operation" AND "suture". The Internet was searched using the Google search engine. A manual search was performed by checking the reference lists of the original reports and the review articles retrieved through the electronic searches to identify studies not yet included in the computerized databases. The final search was carried out on September 1, 2013, without restrictions regarding publication year or language.

\section{Inclusion and exclusion criteria}

The articles were considered eligible if the studies met the following inclusion criteria: (i) study type: RCT; (ii) population: glaucoma patients (but not including secondary glaucoma, congenital glaucoma, or previous intraocular surgery) who failed to respond to conservative therapy; (iii) intervention: trabeculectomies performed with releasable sutures versus trabeculectomy without releasable sutures, with or without the use of antimetabolites; (iv) outcome variables: at least one of the outcomes of interest was included; and (v) follow-up time: $\geq 6$ months. Abstracts from conferences, full texts without raw data available for retrieval, duplicate publications, letters, and reviews were excluded. For publications reporting on the same study population, the article reporting the results of the last endpoint was included, and data that could not be obtained from one publication were obtained from other publications.

\section{Outcome measures}

For efficacy, the primary outcome was the percentage of the IOP reduction (IOPR\%). When authors reported the mean and standard deviation (SD) of the IOPR\%, we used these values directly. For studies that only reported absolute values for the IOP at baseline and at the endpoint, the IOPR and the SD of the IOPR ( $\left.\mathrm{SD}_{\mathrm{IOPR}}\right)$ were calculated as follows: $\mathrm{IOPR}=\mathrm{IOP}_{\text {baseline }}-\mathrm{IOP}_{\text {endpoint }}$, $\mathrm{SD}_{\text {IOPR }}=\left(\mathrm{SD}_{\text {baseline }}^{2}+\mathrm{SD}_{\text {endpoint }}^{2}-\mathrm{SD}_{\text {baseline }} \times \mathrm{SD}_{\text {endpoint }}\right)^{1 / 2}$. The IOPR\% and the SD of the IOPR\% (SDIOPR\%) were estimated by IOPR\% $=\mathrm{IOPR} / \mathrm{IOP}_{\text {baseline }}$ and $\mathrm{SD}_{\mathrm{IOPR} \%}=$ $\mathrm{SD}_{\mathrm{IOPR}} / \mathrm{IOP}_{\text {baseline. }}$.

The secondary outcome measure was the proportion of patients with complete success, which was defined as the target endpoint IOP without antiglaucoma medication, and with qualified success, which was defined as the target endpoint IOP with or without antiglaucoma medications. We assessed tolerability by considering the proportions of patients with postoperative complications, including hypotony, choroidal effusion, flat anterior chambers, hyphema, and cataracts.

\section{Data extraction}

The data were extracted separately by two reviewers (Z.M.W. and W.W.), and they were rechecked after the first extraction. Discrepancies between the two independent data extractions were resolved by discussion. The information extracted from each study included the authors of the study, year of publication, study design, location of the trial, duration of the study, and number of subjects including their age and sex, their use of antimetabolites, their IOP measurements, and their success rates. The number of withdrawals and patients reporting adverse events were also recorded.

\section{Risk of bias assessment}

Two reviewers (Z.M.W. and W.W.) independently assessed the risk of bias in each trial, following the domain-based evaluation as described in the Cochrane Handbook for Systematic Reviews of Interventions 5.1.0 [20]. Two of the authors subjectively reviewed all the studies and assigned a value of "high", "low", or "unclear" to the following factors: (a) selection bias (Was there adequate generation of the randomization sequence? Was allocation concealment satisfactory?); (b) performance and detection bias (Was there blinding of participants, personnel, and outcome assessors?); (c) attrition bias (Were incomplete outcome data sufficiently assessed and dealt with?); (d) reporting bias (Was there evidence of selective outcome reporting?); and (e) other sources of bias (Were any other sources of bias identified?). Any discrepancies 


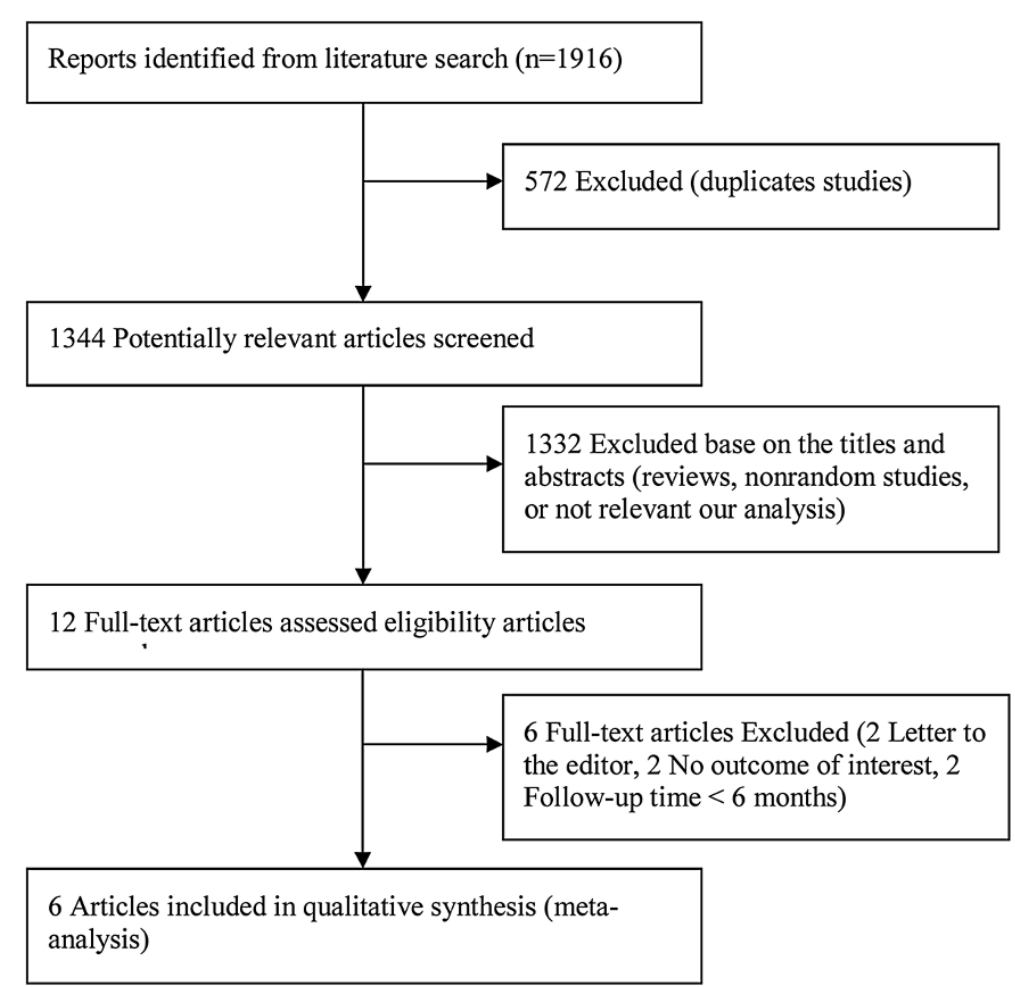

Figure 1 Flow diagram of studies included in this meta-analysis.

between the two authors were discussed, and a consensus was reached.

\section{Statistical analysis}

The outcome measure was assessed on an intent-to-treat (ITT) basis. Given that some of the trials did not report all the outcomes of interest, we conducted a separate metaanalysis for each comparison and outcome. Considering the differences in clinical characteristics among the study groups and the variations in sample size, it was assumed that heterogeneity was present even when no statistical significance was identified. Thus, the data were combined using a random effects model. The weighted mean differ- ence (WMD) of continuous variables and the risk ratio $(R R)$ of dichotomous variables were measured. Both outcomes were reported with a $95 \%$ confidence interval (CI). Statistical heterogeneity among the studies was evaluated with $\chi^{2}$ and $I^{2}$ tests. A subgroup analysis was carried out to evaluate the impact of the surgical characteristics on the results. To detect publication biases, we explored asymmetry in funnel plots. These were examined visually. Furthermore, the Begg and Egger measures were calculated [21,22]. $P<0.05$ was considered statistically significant in the test for an overall effect. The analysis was conducted using the Stata software package (Version 11.0; Stata Corp., College Station, TX).

Table 1 Characteristics of included studies

\begin{tabular}{|c|c|c|c|c|c|c|c|c|c|}
\hline \multirow{2}{*}{ Trial (year) } & \multirow{2}{*}{ Design } & \multirow{2}{*}{ Country } & \multirow{2}{*}{ Eyes* } & \multirow{2}{*}{ Patients* } & \multicolumn{2}{|c|}{ Sex (male/female) } & \multirow{2}{*}{$\begin{array}{l}\text { Age } \\
\text { (year) }\end{array}$} & \multirow{2}{*}{$\begin{array}{l}\text { Follow- } \\
\text { up (mo) }\end{array}$} & \multirow{2}{*}{$\begin{array}{c}\text { Use of } \\
\text { antimetabolite }\end{array}$} \\
\hline & & & & & Without releasable suture & Releasable suture & & & \\
\hline Caporossi(2009) & $\mathrm{RCT}$ & Italy & $33 / 33$ & $33 / 33$ & $21 / 12$ & $17 / 16$ & $61.7 / 66.1$ & $14 / 14$ & MMC \\
\hline Kobayashi(2011) & $\mathrm{RCT}$ & Japan & $25 / 25$ & $25 / 25$ & $12 / 13$ & $14 / 11$ & $69.2 / 69.6$ & $12 / 12$ & MMC \\
\hline Simsek (2005) & $\mathrm{RCT}$ & Turkey & $32 / 32$ & $32 / 32$ & $19 / 13$ & $17 / 15$ & $61.2 / 58.3$ & $11.0 / 11.5$ & no \\
\hline Unlu(2000) & $\mathrm{RCT}$ & Turkey & $20 / 18$ & $18 / 17$ & $9 / 9$ & $9 / 8$ & $60.9 / 61.4$ & 8.3/8.1 & MMC \\
\hline Raina(1998) & $\mathrm{RCT}$ & India & $15 / 15$ & $15 / 15$ & $5 / 10$ & $9 / 6$ & $54.7 / 53.2$ & $12 / 12$ & no \\
\hline Aykan(2007) & $\mathrm{RCT}$ & Turkey & $27 / 21$ & 24/19 & $16 / 11$ & $11 / 10$ & $59.0 / 61.0$ & $6 / 6$ & MMC \\
\hline
\end{tabular}

*Trabeculectomy without releasable suture group/trabeculectomy with releasable suture group.

Abbreviations: $R C T$ prospective randomized controlled trial, MMC mitomycin C. 


\section{Results}

Literature search

A total of 1,916 articles were initially identified. The abstracts were reviewed, and 19 articles with potentially relevant trials were reviewed in their entirety. Subsequently, six articles with full texts that met the inclusion criteria were assessed $[8,11,12,14,18,19]$. Finally, six studies published between 1998 and 2012 were included in the meta-analysis. Figure 1 provides a flow diagram of the search results.

\section{Characteristics and baseline of the included studies}

The trials were conducted in various countries, including Turkey, Japan, India, and Italy. A total of 296 eyes were included in the meta-analysis: 152 eyes were included in the trabeculectomy without releasable sutures group, and 144 eyes were included in the trabeculectomy with releasable sutures group. The mean age ranged from 53.2 years to 69.6 years. The duration of the studies ranged from 6 months to 14 months. The characteristics of the eligible studies are summarized in Table 1.

\section{Risk of bias}

The risk-of-bias analysis (Figures 2 and 3) revealed that only two of the included studies $[18,19]$ adequately reported the randomization protocol and that none of the studies described allocation concealment. Additionally, none of the trials sufficiently described their blinding methods. No selective reporting was evident, as it was clear from the published articles that all the main prespecified outcomes were reported. The majority of the trials had a low risk of bias in the "other sources of bias" domain.

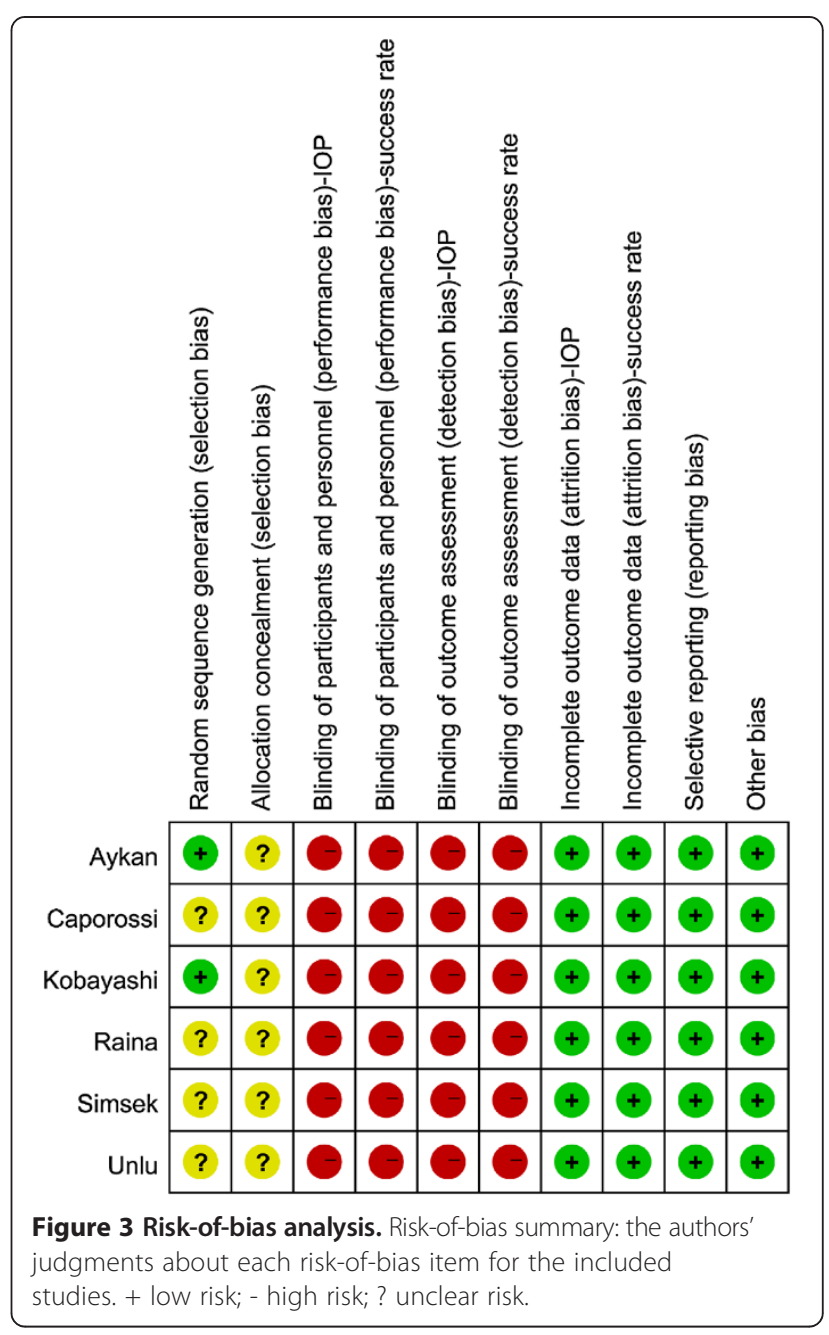

\section{Efficacy analysis}

The IOPR\% of trabeculectomies performed with and without releasable sutures was assessed in the six studies

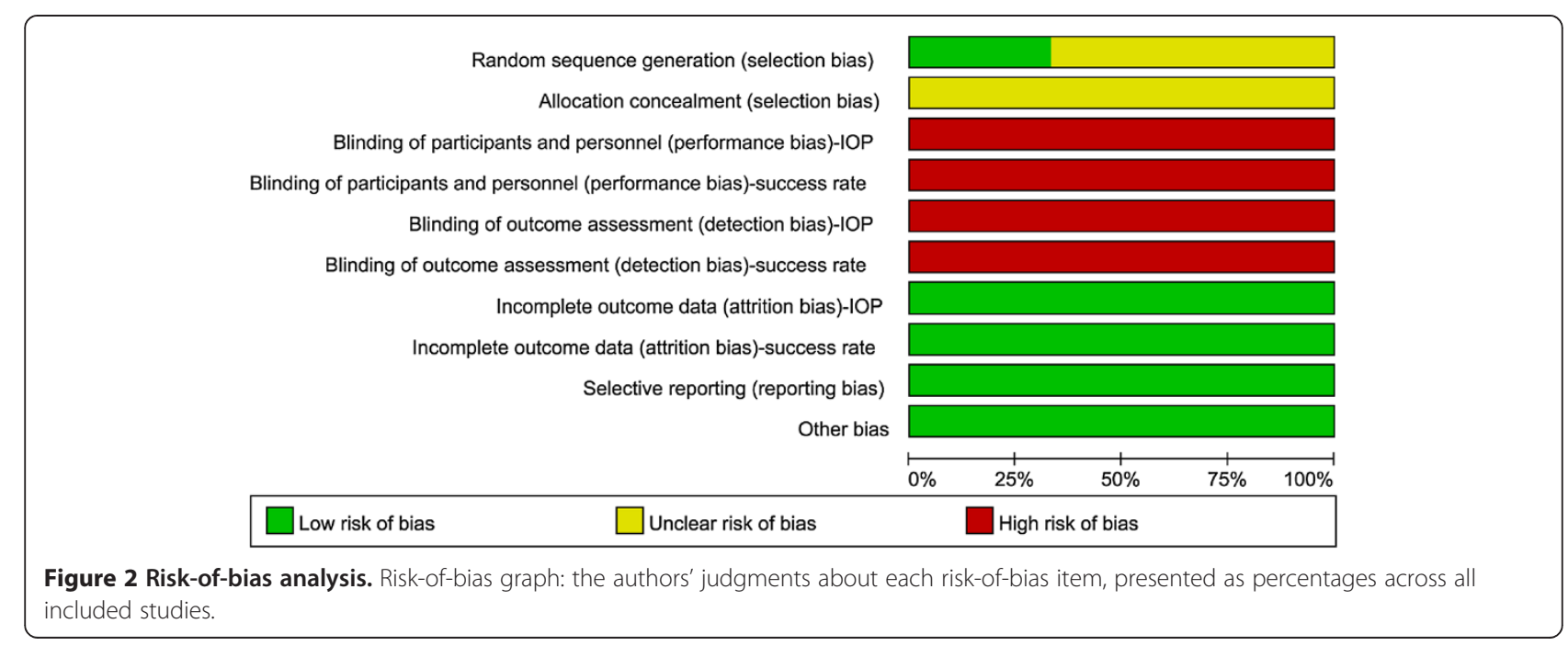


Table 2 Comparison of percentage IOP reduction from baseline between trabeculectomies without releasable suture and trabeculectomies with releasable suture

\begin{tabular}{|c|c|c|c|c|c|}
\hline \multirow[b]{2}{*}{ Trial } & \multicolumn{2}{|c|}{ Without releasable suture } & \multicolumn{2}{|c|}{ Releasable suture } & \multirow{2}{*}{$\begin{array}{l}\text { WMD(random) } \\
(95 \% \mathrm{Cl})\end{array}$} \\
\hline & No. eyes & IOPR (\%) [Mean(SD)] & No. Eyes & IOPR (\%) [Mean(SD)] & \\
\hline Caporossi (2009) & 33 & $30.74(18.88)$ & 33 & $36.59(20.89)$ & $-5.85(-15.46,3.76)$ \\
\hline Kobayashi (2011) & 25 & $49.08(11.43)$ & 25 & $53.96(11.29)$ & $-4.88(-11.18,1.42)$ \\
\hline Unlu (2000) & 20 & $58.20(23.00)$ & 18 & $57.98(29.71)$ & $0.22(-16.81,17.25)$ \\
\hline Raina (1998) & 15 & $36.90(23.25)$ & 15 & $47.35(30.96)$ & $-10.45(-30.04,9.14)$ \\
\hline Aykan (2007) & 27 & $57.69(33.01)$ & 21 & $56.47(25.87)$ & $1.22(-15.44,17.88)$ \\
\hline Total & 120 & & 112 & & $-4.56(-9.24,0.12)$ \\
\hline
\end{tabular}

Test for heterogeneity $X^{2}=1.19, d f=4, P=0.879$.

Test for overall effect $\quad z=1.91, P=0.056$.

Abbreviations: IOP intraocular pressure, $\mathrm{Cl}$ confidence interval, IOPR\% percentage intraocular pressure reduction, WMD weighted mean difference.

involving 296 eyes. The test for heterogeneity between the studies was insignificant $\left(\chi^{2}=1.19 ; P=0.879, I^{2}=0.0 \%\right)$. The combined results showed that both surgical procedures significantly decreased the IOP. The IOPR\% of the trabeculectomies with releasable sutures were numerically greater compared with the baseline. However, the differences in the IOPR\% were not all statistically significant (WMD $=-4.56[-9.24,0.12]$; Table 2). In the subgroup analysis of the use of antimetabolites, the difference between the groups was not statistically significant (Table 3), and there was no statistical heterogeneity between the studies. The funnel plot is shown in Figure 4. All study outcomes were within the 95\% CI, and they were distributed symmetrically, showing no evidence of publication bias. Moreover, neither Begg's test $(P=0.806)$ nor Egger's test $(P=0.661)$ suggested publication bias in these trials.

Of the five studies that reported the probability of complete success, no significant difference was found between the two groups (pooled $R R=0.92$ [0.80, 1.05]; Table 4). The assessment of publication bias using Begg's test $(P=0.806)$ and Egger's test $(P=0.822)$ showed that no publication bias existed among the included trials. Additionally, the difference in the qualified success rate between the two groups was not statistically significant (pooled $R R=0.99[0.89,1.11]$; Table 4). Publication bias in this outcome was not assessed because of the limited number of studies included.

\section{Tolerability analysis}

A comparison of adverse events between the RCTs of the trabeculectomies with and without releasable sutures is presented in Table 5. Hypotony, a flat anterior chamber, choroidal effusion, hyphema, and cataract formation were the commonly reported postoperative complications. Trabeculectomy without releasable sutures was associated with a significantly higher frequency of hypotony and a flat anterior chamber than trabeculectomy with releasable sutures, with pooled RRs of 4.04 (range 1.88-8.68) and 2.57 (range 1.25-5.30), respectively. However, no significant differences in the incidence of choroidal effusion, hyphema, and cataract formation were found between the two groups, with pooled ORs of 2.51 (range 0.89-7.07), 0.93 (range $0.43-2.03$ ), and 1.59 (range $0.48-5.30$ ), respectively.

\section{Discussion}

Trabeculectomy is still considered the mainstay for medically uncontrolled glaucoma [23]. Several modifications and variations have been developed to maximize the benefits of treatment while minimizing adverse events [19,23,24]. However, this technique is associated with complications. Trabeculectomy supplemented with releasable sutures can decrease the IOP rate and reduce post-trabeculectomy complications during the early postoperative period [14].

Previous studies have prospectively evaluated the efficacy and safety of trabeculectomies performed with and without releasable sutures $[8,11,12,14,18,19]$. All the trials showed

Table 3 Subgroup analyses of percentage reduction in IOP

\begin{tabular}{|c|c|c|c|c|c|c|c|}
\hline \multirow[b]{2}{*}{ Subgroup } & \multirow[b]{2}{*}{ Studies (n) } & \multirow{2}{*}{$\begin{array}{l}\text { WMD (Fixed) } \\
(95 \% \mathrm{Cl})\end{array}$} & \multicolumn{3}{|c|}{ Heterogeneity } & \multicolumn{2}{|c|}{ Overall effect } \\
\hline & & & Q & $P$ & $I^{2}(\%)$ & Z & $P$ \\
\hline All trials & 5 & $-4.56(-9.24,0.12)$ & 1.19 & 0.879 & $0.00 \%$ & 1.91 & 0.056 \\
\hline \multicolumn{8}{|c|}{ Use of antimetabolite } \\
\hline Yes & 4 & $-4.21(-9.02,0.61)$ & 0.82 & 0.844 & $0.00 \%$ & 1.71 & 0.087 \\
\hline No & 1 & $-4.93(-9.61,-0.26)$ & 0.00 & - & - & 1.70 & 0.090 \\
\hline
\end{tabular}




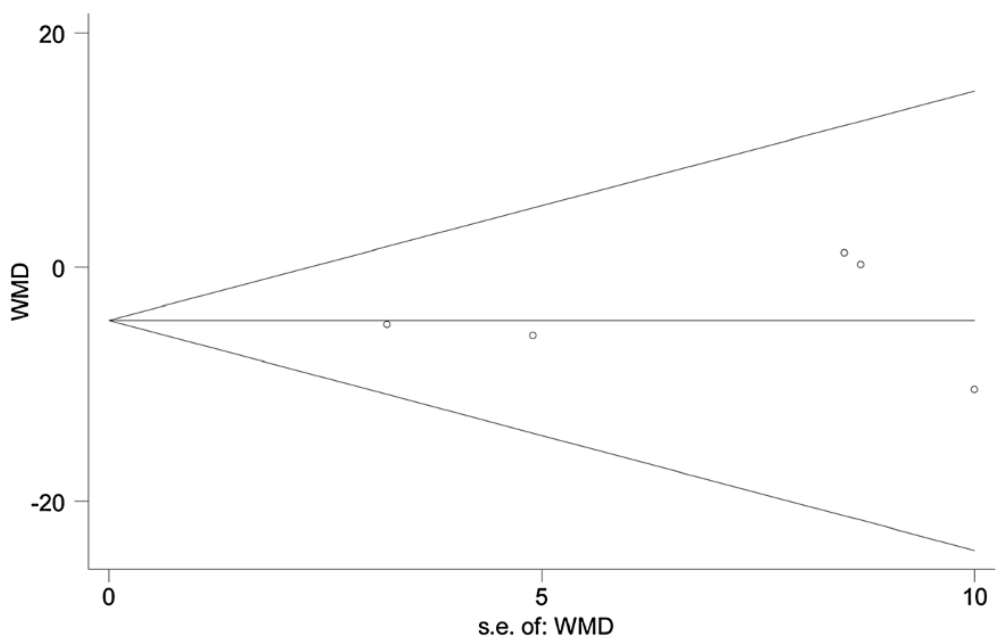

Figure 4 Tests for publication bias for WMD of the percentage of IOP reduction (IOPR\%).

that the techniques were comparably efficacious in lowering the IOP. However, the various trials showed different incidence rates of complications, and some trials included only small sample sizes, which hindered the ability to draw conclusions for clinical practice. Therefore, the present meta-analysis was undertaken to assess the efficacy and tolerability of both surgical procedures in the treatment of uncontrolled glaucoma.

To the best of our knowledge, this is the first metaanalysis to explore the efficacy and tolerability of trabeculectomy performed with the releasable suture procedure. We reviewed six RCTs of trabeculectomies, with and without releasable sutures in patients with uncontrolled glaucoma. The pooled results from the meta-analysis, using a random effects model, suggested that an intraoperative releasable suture application is comparable to trabeculectomies without releasable sutures in lowering IOP and that it is comparable to trabeculectomies without releasable sutures in the complete and qualified success rates. Furthermore, the results of the subgroup analyses were quite similar and robust.

This releasable suture surgical procedure was developed as an alternative to trabeculectomy without releasable sutures, mainly to overcome possible complications, such as hypotony, a shallow, flat anterior chamber, and choroidal detachment. We found that the releasable suture surgical procedure reduced these complications when compared with the traditional practice, with a statistically significant difference in the incidence of hypotony and flat anterior chambers between the two groups. Inadequate tension in the scleral sutures was responsible for the majority of cases with flat anterior chambers and hypotony in an otherwise uneventful trabeculectomy [25]. The lower incidence of complications in the group with releasable sutures was mainly due to the relatively tight sclera flap releasable sutures, which can control the aqueous outflow more easily. The current study provides interesting findings that could be useful in the selection of surgical procedures.

The first strength of the present analysis is that it focused on a direct comparison between trabeculectomies performed with and without releasable sutures, rather than on an indirect comparison. Second, the likelihood of bias was minimized by developing a detailed protocol before initiating the study, by performing a meticulous search for published studies, and by using explicit methods for study selection, data extraction, quality assessment, and

Table 4 Comparison of complete success and qualified success between trabeculectomies without releasable suture and trabeculectomies with releasable suture

\begin{tabular}{|c|c|c|c|c|c|c|c|c|c|}
\hline \multirow[b]{2}{*}{ Trial } & \multirow[b]{2}{*}{ Studies ( $n$ ) } & \multicolumn{2}{|c|}{ Success rate, n/N(\%) } & \multirow[b]{2}{*}{ RR $(95 \% \mathrm{Cl})$} & \multicolumn{3}{|c|}{ Heterogeneity } & \multicolumn{2}{|c|}{ Overall effect } \\
\hline & & Without releasable suture & Releasable suture & & Q & $P$ & $\mathrm{I}^{2}(\%)$ & $\mathrm{z}$ & $P$ \\
\hline \multicolumn{10}{|c|}{ Complete success } \\
\hline All trials & 5 & 90/119 (75.63\%) & 92/111 (82.88\%) & $0.92(0.80,1.04)$ & 1.31 & 0.860 & $0.00 \%$ & 1.31 & 0.191 \\
\hline \multicolumn{10}{|c|}{ Qualified success } \\
\hline All trials & 4 & 83/87 (95.40\%) & 76/79 (96.20\%) & $0.99(0.89,1.11)$ & 0.34 & 0.560 & $0.00 \%$ & 0.20 & 0.845 \\
\hline
\end{tabular}


Table 5 Comparison of adverse events between trabeculectomies without releasable suture and trabeculectomies with releasable suture

\begin{tabular}{|c|c|c|c|c|c|c|c|c|c|}
\hline \multirow[b]{2}{*}{ Adverse events } & \multirow[b]{2}{*}{ Studies (n) } & \multicolumn{2}{|c|}{ Crude event rate, $n / N$} & \multirow[b]{2}{*}{ RR $(95 \%$ Cl) } & \multicolumn{3}{|c|}{ Heterogeneity } & \multicolumn{2}{|c|}{ Overall effect } \\
\hline & & Without releasable suture & Releasable suture & & Q & $P$ & $\overline{I^{2}(\%)}$ & Z & $P$ \\
\hline Hypotony & 3 & $28 / 72$ & $6 / 72$ & $4.04(1.88,8.68)$ & 1.02 & 0.602 & $0.00 \%$ & 3.58 & $<0.001$ \\
\hline Flat anterior chamber & 5 & $37 / 137$ & $12 / 129$ & $2.57(1.25,5.30)$ & 5.35 & 0.254 & $25.20 \%$ & 2.56 & 0.010 \\
\hline Choroidal effusion & 4 & $10 / 93$ & $3 / 91$ & $2.51(0.89,7.07)$ & 0.04 & 0.998 & $0.00 \%$ & 1.74 & 0.082 \\
\hline Hyphema & 6 & $12 / 152$ & $11 / 144$ & $0.93(0.43,2.03)$ & 3.95 & 0.557 & $0.00 \%$ & 0.18 & 0.855 \\
\hline Cataract formation & 3 & $7 / 62$ & $4 / 54$ & $1.59(0.48,5.30)$ & 0.58 & 0.750 & $0.00 \%$ & 0.75 & 0.451 \\
\hline
\end{tabular}

Abbreviations: $\mathrm{RR}$ risk ratio, $\mathrm{Cl}$ confidence interval.

statistical analysis. Finally, the subgroup analysis demonstrated that the conclusions of this analysis are robust.

One major limitation of this analysis is that we cannot fully exclude publication bias. To avoid publication bias, we conducted not only an electronic search but also a manual search to identify all potentially relevant articles, including those that were published or nonpublished. Unfortunately, it is possible that we failed to include some papers, especially those published in other languages. The second limitation is that although no significant heterogeneity was found, the studies were carried out with small or very small sample sizes, inadequate allocation concealment, and inadequate or no double-blinding. These factors can affect the interpretation of the results. The third limitation is that the analyses of clinically relevant outcome measures were based on data pooled from trials with different follow-up periods. To decrease this bias, we included only studies with follow-up times of at least 6 months. Another potential source of heterogeneity in the results was the assessment criterion for success. Success was defined as a target endpoint IOP. Although such assessments of success are widely used as outcome measures in clinical trials, further research is still needed to determine their validity, reliability, and sensitivity to ensure that the best is chosen. Finally, with regard to the complication of cataract formation, the included studies seldom mentioned the cataract grading. Cataract grading following trabeculectomies performed with and without releasable sutures should be the focus of future studies.

\section{Conclusions}

In conclusion, the results of this meta-analysis of six RCTs indicated that trabeculectomies with releasable sutures showed equivalent efficacy to trabeculectomies without releasable sutures in controlling the IOP and that the proportions of patients in both groups who achieved the target IOP were comparable. The analysis of the incidence of postoperative complications showed that a trabeculectomy with releasable sutures is safer than a trabeculectomy without releasable sutures.
Competing interests

The authors declare that they have no competing interests.

\section{Authors' contributions}

All authors conceived of and designed the experimental protocol. MZ and WW collected the data. All authors were involved in the analysis. MZ wrote the first draft of the manuscript. $M Z, W W$ and $X Z$ reviewed and revised the manuscript and produced the final version. All authors read and approved the final manuscript.

\section{Acknowledgements}

Supports: This research was supported by the National Natural Science Foundation of China (81170849), and Guangdong Provincial Natural Science Foundation (S2011020002401).

Received: 15 October 2013 Accepted: 28 March 2014

Published: 31 March 2014

\section{References}

1. Bertrand V, Fieuws S, Stalmans I, Zeyen T: Rates of visual field loss before and after trabeculectomy. Acta Ophthalmol 2014, 92:116-120.

2. Kirwan JF, Lockwood AJ, Shah P, Macleod A, Broadway DC, King AJ, McNaught Al, Agrawal P: Trabeculectomy in the 21st century: a multicenter analysis. Ophthalmol 2013, 120:2532-2539.

3. Back M: Trabeculectomy for glaucoma. Arch Ophthalmol 1975, 93:1372.

4. Dreyer EB: Post-trabeculectomy hypotony. Ophthalmo/ 1997, 104:1367.

5. Alemu B: Trabeculectomy: complications and success in IOP control. Ethiop Med J 1997, 35:1-11.

6. Berke SJ, Bellows AR, Shingleton BJ, Richter CU, Hutchinson BT: Chronic and recurrent choroidal detachment after glaucoma filtering surgery. Ophthalmol 1987, 94:154-162.

7. Prevezas DC, Melissurgos ID: Adjustable temporal suture in trabeculectomy with scleral flap. Ophthalmic Surg Lasers 1997, 28:745-750.

8. Raina UK, Tuli D: Trabeculectomy with releasable sutures: a prospective, randomized pilot study. Arch Ophthalmol 1998, 116:1288-1293.

9. Kolker AE, Kass MA, Rait JL: Trabeculectomy with releasable sutures. Arch Ophthalmol 1994, 112:62-66.

10. Matlach J, Hoffmann N, Freiberg FJ, Grehn F, Klink T: Comparative study of trabeculectomy using single sutures versus releasable sutures. Clin Ophthalmol 2012, 6:1019-1027.

11. Caporossi A, Balestrazzi A, Malandrini A, Tosi GM, Caporossi T, Frezzotti P, Lomurno L: A randomized prospective study comparing trabeculectomy with and without the use of a new removable suture. Int Ophthalmol 2009, 29:359-365.

12. Simsek T, Citirik M, Batman A, Mutevelli S, Zilelioglu O: Efficacy and complications of releasable suture trabeculectomy and standard trabeculectomy. Int Ophthalmol 2005, 26:9-14.

13. Liang YB, Feng MY, Meng HL, Fan SJ, Wang X, Xie LL, Yi P, Tang X, Wang NL, Thomas R: Early efficacy and complications of releasable sutures for trabeculectomy in primary angle-closure glaucoma: a randomized clinical trial. J Glaucoma 2014, 23:136-141.

14. Unlu K, Aksunger A, Soker S, Ertem M: Mitomycin C primary trabeculectomy with releasable sutures in primary glaucoma. Jpn J Ophthalmol 2000, 44:524-529. 
15. Shaffer RN, Hetherington JJ, Hoskins HJ: Guarded thermal sclerostomy. Am J Ophthalmol 1971, 72:769-772.

16. Savage JA, Condon GP, Lytle RA, Simmons RJ: Laser suture lysis after trabeculectomy. Ophthalmol 1988, 95:1631-1638.

17. L'Esperance FJ: The ocular histopathologic effect of krypton and argon laser radiation. Am J Ophthalmol 1969, 68:263-273.

18. Kobayashi H, Kobayashi K: A comparison of the intraocular pressure lowering effect of adjustable suture versus laser suture lysis for trabeculectomy. J Glaucoma 2011, 20:228-233.

19. Aykan U, Bilge AH, Akin T, Certel I, Bayer A: Laser suture lysis or releasable sutures after trabeculectomy. J Glaucoma 2007, 16:240-245.

20. Higgins J, Green S: Cochrane Handbook for Systematic Reviews of Interventions Version 5.1.0.: The Cochrane Collaboration. 2011. Available: www.cochrane-handbook.org.

21. Egger M, Davey SG, Schneider M, Minder C: Bias in meta-analysis detected by a simple, graphical test. BMJ 1997, 315:629-634.

22. Begg $C B$, Mazumdar M: Operating characteristics of a rank correlation test for publication bias. Biometrics 1994, 50:1088-1101.

23. Wells AP, Bunce C, Khaw PT: Flap and suture manipulation after trabeculectomy with adjustable sutures: titration of flow and intraocular pressure in guarded filtration surgery. J Glaucoma 2004, 13:400-406.

24. Jones $\mathrm{E}$, Clarke J, Khaw PT: Recent advances in trabeculectomy technique. Curr Opin Ophthalmol 2005, 16:107-113.

25. Watkins PJ, Brubaker RF: Comparison of partial-thickness and full-thickness filtration procedures in open-angle glaucoma. Am J Ophthalmol 1978, 86:756-761.

doi:10.1186/1471-2415-14-41

Cite this article as: Zhou et al:: Trabeculectomy with versus without releasable sutures for glaucoma: a meta-analysis of randomized controlled trials. BMC Ophthalmology 2014 14:41.

\section{Submit your next manuscript to BioMed Central and take full advantage of:}

- Convenient online submission

- Thorough peer review

- No space constraints or color figure charges

- Immediate publication on acceptance

- Inclusion in PubMed, CAS, Scopus and Google Scholar

- Research which is freely available for redistribution 